\title{
S Research Square

\section{Growth in Ever-Increasing Acidity Condition Enhanced the Adaption and Bioleaching of Leptospirlium Ferriphilum}

Ronghui Liu ( $\nabla$ rhliu959@sina.cn )

Southern University of Science and Technology https://orcid.org/0000-0002-9755-854X Hongbo Zhou

Central South University

\section{Research Article}

Keywords: Leptospirilum ferriphilum, bioleaching, low pH, adaptation

Posted Date: May 26th, 2021

DOl: https://doi.org/10.21203/rs.3.rs-528164/v1

License: (c) (1) This work is licensed under a Creative Commons Attribution 4.0 International License.

Read Full License 


\section{Abstract}

Low $\mathrm{pH}$ could eliminate the jarosite accumulation and improve the interfacial reaction rate during bioleaching process. However, a great challenge existed between microbial activity and bioleaching ability in low pH conditions. This study demonstrated that the adaption and bioleaching of Leptospirilum ferriphilum could be improved after long term adaptive evolution under acidity condition. It was found that the acidity adapted strain showed robust activity in wider $\mathrm{pH}$, high concentration of ferrous iron and lower temperature. Although the enhancement for heavy metal tolerance was limited, the tolerance for $\mathrm{MgSO}_{4}, \mathrm{Na}_{2} \mathrm{SO}_{4}$ and organic matter was great. More importantly, both pyrite and printed circuit boards bioleaching revealed the higher bioleaching ability of the acid-resistant strain. These adaptation and bioleaching details provided an available approach for the improvement of bioleaching techniques.

\section{Introduction}

Acidophiles were the most efficient community on the metals bioleaching from sulfide minerals. Recent disclosures demonstrated that these microorganisms still conducted crucial function for metals extraction from solid waste such as waste printed wiring board (Xia et al., 2017), electroplating sludge (Zhou et al., 2019) and coal fly ash (Fan et al., 2019). However, the fickle bioleaching environment such as fluctuated $\mathrm{pH}$ and the increased metal ion was detrimental to the growth of microorganisms and thus weakened the biological effects, which resulted in low leaching efficiency.

In order to enhance the bioleaching efficiency, our previous studies developed an extreme acid tolerance consortium through three steps adaptive evolution (Liu et al., 2019b) and subsequent research revealed that the obtained consortia could extract near $100 \%$ metals from waste resin powder at high pulp density (Liu et al., 2020). For adaptation, the synergetic interaction in this consortium plays an important role in the stable maintenance of function in wider conditions (Liu et al., 2019b). However, the adaptive evolutionary community on the effect of a single microorganism for environmental adaptations is unknown. An in-depth analysis of these problems can not only deepen the understanding of the characteristics of the adaptive evolutionary communities but also contribute to the further development and utilization of adaptive evolutionary strains.

In this study, the main strain in the extreme acid tolerance community was isolated with a low $\mathrm{pH}$ gradient dilution method. Comparative investigation of original and adapted strain on growth at wider $\mathrm{pH}$, temperature, concentration of metal ion, $9 \mathrm{~K}$ basic salt and organic matters were explored. After that, the bioleaching capacity of isolated strain in wider conditions was illustrated. These findings preliminary revealed the strain evolution characteristics of adaptation and provide some new insight to the adaptive evolution of acidophiles.

\section{Materials And Methods}

\subsection{Medium and strains}


Leptospirillum ferriphilum DX-m was isolated from the main bio-heap of Dexing copper mine and reserved by our laboratory. The extreme acid adaptive community used in this study was obtained through a modified adaptive evolution process by our laboratory (Liu et al., 2019b). The medium used for the culture of community and pure culture was described as previous reports (Liu et al., 2019b; Liu et al., 2019c).

\subsection{Isolation of the evolved strain}

Previous studies revealed the extreme acid adaptive community mainly composed of Sulfobacillus spp., Leptospirillum spp. and Ferroplasma spp.(Liu et al., 2019b). During isolating process, consideration of the indispensability of organic matter for the quick growth of both Sulfobacillus spp. and Ferroplasma spp.(Zhang et al., 2015; Zhou et al., 2015), selective liquid medium without the addition of organic matter was used to achieve the separation of $L$. ferriphilum. In order to prevent the interference of some nonacid-resistant mutants or reverting mutants, a low pH strategy was employed during the gradient dilution process. Simply, the obtained adaptive evolutionary community was inoculated into a shaking flask containing a selective medium ( $9 \mathrm{~K}$ inorganic salt, $350 \mathrm{mM} \mathrm{Fe}^{2+}$ and $\mathrm{pH} 0.7$ ) after gradient dilution. After $85 \%$ ferrous iron was oxidized to ferric iron, cells were collected by centrifugation and DNA was extracted by the DNA extraction kit for further molecular identification by PCR and $16 \mathrm{~S}$ sequencing. The common and specific primers used in this study were same as previous studies (Liu et al., 2019b; Ma et al., 2018).

\subsection{Growth characteristic of starting and evolved strains on different biochemical parameters conditions}

The effects of biochemical parameters on the growth of microorganisms are extremely important for the application of acidophiles (Shiers et al., 2016). This experiment explored effects of ferrous iron concentration $\left(5-30 \mathrm{gL}^{-1}\right), \mathrm{pH}(0.6-1.8)$, temperature $\left(25-45^{\circ} \mathrm{C}\right)$ and $9 \mathrm{~K}$ basic salt $\left(\left(\mathrm{NH}_{4}\right)_{2} \mathrm{SO}_{4}: 05-3 \mathrm{gL}^{-1}\right.$; $\left.\mathrm{K}_{2} \mathrm{HPO}_{4}: 0.05-0.5 \mathrm{gL}^{-1} ; \mathrm{KCl}: 0-0.01 \mathrm{gL}^{-1}\right)$ on the growth of starting strain and evolved strain under high and low $\mathrm{pH}$ condition. During the experiment, samples were taken at an interval to determine the iron oxidation rate and biomass.

\subsection{Tolerance experiment of starting strain and evolved strain}

During the solid waste leaching process, heavy metals, magnesium ions, sodium ions and organic matter generally increased significantly (Liu et al., 2020), which notably affected the bioleaching efficiency. The tolerance of microorganisms to these factors becomes the key to accelerate metals extraction. This study explored different concentrations of magnesium sulfate $\left(1-20 \mathrm{gL}^{-1}\right)$, sodium sulfate $\left(1-20 \mathrm{gL}^{-1}\right)$ and yeast extract (0-1 $\left.\mathrm{gL}^{-1}\right)$ on the effects of iron oxidation and biomass production of starting and evolved strains. As for tolerance test of heavy metals, 1 and $5 \mathrm{~g} \mathrm{~L}^{-1} \mathrm{Ni} \square \mathrm{Cr} \square \mathrm{Cu}$ and $\mathrm{Co}$ was adopted. The medium used in these assays was $9 \mathrm{~K}$ medium (initial ferrous ion $10 \mathrm{gL}^{-1}$ ), and the culture conditions were $45^{\circ} \mathrm{C}$ and 180 rpm. During the experiment, samples were taken at an interval to determine the changes in ferrous ions. After complete oxidation, the average iron oxidation rate was calculated and the biomass was measured. All experiments were performed in triplicates. 


\subsection{Bioleaching tests}

Bioleaching of waste printed circuit boards and pyrite at different conditions was carried out to evaluate the leaching efficiency of evolved strains. The minerals used in this were present as our previous description (Liu et al., 2019a; Liu et al., 2019b; Liu et al., 2020). Bioleaching experiments were performed in shake flask, and the factors of $\mathrm{pH}(1.6$ and 1.0$)$ and temperature $\left(30^{\circ} \mathrm{C}\right.$ and $\left.45^{\circ} \mathrm{C}\right)$ was taken into consideration. Pyrite bioleaching assays were present as following: $2 \%$ pulp density, 10 days. For PCB bioleaching, the condition was $10 \%$ pulp density, $1 \mathrm{~g} / \mathrm{L}$ ferrous iron and 3 days. The $9 \mathrm{~K}$ basic salt medium and $10 \%$ bacterial inoculation were performed in these experiments. During bioleaching process, bioleaching tests with starting strain were set as the control, and samples were taken at an interval to determine the change in chemical parameters.

\subsection{Analytical methods}

Total cells in the supernatant were determined by direct counts (blood cells counting chambers: XB-K-25, QiuJing, Shanghai, China; Microscope: BX-41TF, Olympus, Tokyo, Japan). The total iron and ferrous iron were determined by a spectrophotometer with the o-phenanthroline (TGI, Shanghai, China) spectrophotometry assay (1500, Thermo, USA). The Cu contents in solutions were analyzed using an inductively coupled plasma-optical emission spectrometer (ICP-OES) (Optima 5300 DV, PerkinElmer Instrument). The $\mathrm{pH}$ of the supernatant was measured with a pH meter (PH-3S) (pHS-3C, Leici, Shanghai, China). The data analysis was performed by SPSS version 24.0 (SPSS Inc., Chicago, IL, USA).

\section{Results And Discussions}

\subsection{Characteristic of acid tolerance strain}

Analysis of the $16 \mathrm{~S}$ rDNA sequences and construction of the phylogenetic tree based on neighbor-joining analysis were performed for molecular identification of isolated strains. The partial 16S rDNA sequence of isolated strains displayed $100 \%$ identity with corresponding sequences of Leptospirilum ferriphilum DX-m (Fig. 1). And the isolated strain was therefore named Leptospirilum ferriphilum DX-m-ALE. Surface groups analysis based on FTIR revealed that the peak intensity in $1080 \mathrm{~cm}^{-1} \otimes 1545 \mathrm{~cm}^{-1} \otimes 1640 \mathrm{~cm}^{-1}$ and $2080 \mathrm{~cm}^{-1}$ increased, while in $1190 \mathrm{~cm}^{-1} \otimes 1290 \mathrm{~cm}^{-1} \otimes 1470 \mathrm{~cm}^{-1} \otimes 1570 \mathrm{~cm}^{-1}$ and $3000 \mathrm{~cm}^{-1}$ decreased (Fig.

2). These data indicating more polysaccharide like substances, $-\mathrm{C}-\mathrm{O}-\mathrm{C}-,-\mathrm{C}=\mathrm{C}-$ and heterocyclic compound in Leptospirilum ferriphilum DX-m-ALE.

\subsection{Iron oxidation and biomass production of Leptospirilum ferriphilum DX-m-ALE in wider conditions}

As illustrated in Fig 3A, the iron oxidation capacity of Leptospirilum ferriphilum DX-m gradually decreased with the decrease of $\mathrm{pH}$ value and no biological iron ion oxidation was observed at $\mathrm{pH} 0.6$. These results indicated serious inhibition of acid on the iron oxidation of Leptospirilum ferriphilum (Zhang et al., 2010). In comparison, Leptospirilum ferriphilum DX-m-ALE completely oxidized $10 \mathrm{~g} / \mathrm{L}$ iron within 48 hours in the range of $\mathrm{pH}$ 0.6-1.8. Meanwhile, the production of biomass showed the same trend with ferrous iron 
oxidation (Fig.3B). These results indicating that the acid resistance of the evolved strain Leptospirilum ferriphilum DX-m-ALE was improved.

The effect of temperature on iron oxidation of both the starting strain Leptospirilum ferriphilum DX-m and the evolving strain Leptospirilum ferriphilum DX-m-ALE was presented in Fig 3C. In the case of pH 1.6, the iron oxidation rate for the evolved strain was $250 \mathrm{mg} \mathrm{L}^{-1} \mathrm{~h}^{-1}$ at $30^{\circ} \mathrm{C}$, while that of the starting strain was $80 \mathrm{mg} \mathrm{L}^{-1} \mathrm{~h}^{-1}$. Even the temperature was at $25^{\circ} \mathrm{C}$, the evolved strain showed $150 \mathrm{mg} \mathrm{L}^{-1} \mathrm{~h}^{-1}$ iron oxidation rate. It is obvious that the evolved strain had stronger adaptability at low temperature. In the case of $\mathrm{pH}$ 0.7 , the evolved strain showed the maximum iron oxidation rate of $350 \mathrm{mgL}^{-1} \mathrm{~h}^{-1}$ at $45^{\circ} \mathrm{C}$. However, the iron oxidation rate in low $\mathrm{pH}$ culture was slightly lower than that in high $\mathrm{pH}$ culture when $\mathrm{pH}$ below $37^{\circ} \mathrm{C}$. For biomass production, the lower the temperature, the less biomass. It is not hard to conclude that the adaptive evolutionary strain Leptospirilum ferriphilum DX-m-ALE showed a wider range of temperature adaptability and could maintain this superiority under the low $\mathrm{pH}$ environment, but the double stress of low temperature and acidity still affects the activity of the evolved strain.

The combined effect of ferrous iron and $\mathrm{pH}$ on the iron oxidation for Leptospirilum ferriphilum DX-m-ALE and Leptospirilum ferriphilum DX-m was also determined (Fig 3D-F). At pH 1.6, both strains oxidized all ferrous iron within 48 hours below 5-10 g/ $\mathrm{L} \mathrm{Fe}^{2+}$. However, high concentrations of ferrous iron seriously inhibit the growth of starting strain. In the case of $30 \mathrm{~g} / \mathrm{L} \mathrm{Fe}^{2+}$, little ferrous iron was observed to be biologically oxidized with 72 hours. In contrast, the evolved strain completely oxidized ferrous iron to ferric iron. These results demonstrated the evolved strain has a greater tolerance to $\mathrm{Fe}^{3+}$ compared to the starting strain. At $\mathrm{pH} 0.7$, the evolved strain showed higher iron oxidation ability when the ferrous iron concentration below $20 \mathrm{~g} / \mathrm{L}$, which may due to Leptospirilum ferriphilum under low pH need more energy to resist acid stress (Matsumoto et al., 2004). However, dual stress of acid and high concentration of iron ions still inhibited the iron oxidation function of the evolved strain Leptospirilum ferriphilum DX-m-ALE, the time to oxidize $30 \mathrm{gL}^{-1} \mathrm{Fe}^{2+}$ extended to 84 hours. Based on the above results, it is concluded that Leptospirilum ferriphilum DX-m-ALE showed greater capacity for ferrous iron oxidation under different conditions

\subsection{The adaptation for the basic salt in $9 \mathrm{~K}$ medium}

During the bioleaching process, the constituent lack of $9 \mathrm{~K}$ basic salt seriously inhibited the growth of microorganisms, but the excessive chemicals in 9Kmeidum not only caused energy waste but also lead to the production of secondary precipitates and limited the growth of cells (Gramp et al., 2008). The reasonable component and concentration of basic salt is allimportant for the application of bioleaching techniques. It was observed that the iron oxidation capacity and biomass production of the evolved Leptospirilum ferriphilum in different concentration of basic salt was different from the starting strain.

Little influence on the iron oxidation rate of the starting strain was observed at 1-3 g/ $\mathrm{L}$ ammonium salt, and the high concentration of ammonium salt may not be beneficial to the growth of starting strain (Fig. 4A). In comparison, the evolved strain showed a higher iron oxidation rate in the wider concentration of 
ammonium sulfate. In addition, the culture of the evolved L. ferriphilum in the low $\mathrm{pH}$ condition showed similar trends. These results indicated little dependency on ammonium salt concentration for starting strain and evolved strain, but the evolved strain showed higher growth capacity under different ammonium salt concentrations.

The negligible effect of $\mathrm{KCl}$ on iron oxidation rate was observed for both evolved and starting strains (Fig. 4B). Even in the absence of potassium chloride, the iron oxidation rate achieved for $250 \mathrm{mgL}^{-1} \mathrm{~h}^{-1}$. It was noted that the high concentration of potassium chloride improved the biomass production. Particularly in the case of $\mathrm{pH}$ 0.7, the higher concentration of potassium chloride, the more biomass was detected. This is possible that a higher concentration potassium ion could improve the acid resistance stress of microorganisms(Guan and Liu, 2020). It is demonstrated that the evolved strain could adapt wide range of $\mathrm{KCl}$ and thus would improve the growth.

The concentration of $\mathrm{K}_{2} \mathrm{HPO}_{4}$ had a great influence on iron oxidation and biomass of strains, and the effect of $\mathrm{K}_{2} \mathrm{HPO}_{4}$ on the iron oxidation capacity for starting strain and evolution was similar (Fig 4C). The maximum iron oxidation rate and biomass was obtained at $0.2 \mathrm{~g} / \mathrm{L} \mathrm{K}_{2} \mathrm{HPO}_{4}$. However, the evolved strain showed higher iron oxidation rate at $\mathrm{pH}$ 0.7. And the biomass gradually increased with the increase of the concentration of $\mathrm{K}_{2} \mathrm{HPO}_{4}$. This may because more potassium ions improved the acidity resistance of microorganisms (Baker-Austin and Dopson, 2007) and low pH eliminated the inhibition effect of jarosite on cells. Therefore, the $0.2 \mathrm{~g} \mathrm{~L}^{-1} \mathrm{~K}_{2} \mathrm{HPO}_{4}$ is more suitable for the culture of the strain at high $\mathrm{pH}$, but the increase of $\mathrm{K}_{2} \mathrm{HPO}_{4}$ concentration at low $\mathrm{pH}$ is beneficial to increase the growth rate of microorganisms. In all, the evolved strain could grow in a wider range of $9 \mathrm{~K}$ basic salt.

\subsection{The tolerance for high concentration salts, organic matter and heavy metals}

After acid adaptation, L. ferriphilum showed the improved tolerance for higher concentrations of magnesium sulfate (Fig.5A). And the average iron oxidation rate of evolved L. ferriphilum reached 341 $\mathrm{mg} \mathrm{L}^{-1} \mathrm{~h}^{-1}$ under $10 \mathrm{~g} / \mathrm{L} \mathrm{MgSO}_{4}$ condition. Even at $20 \mathrm{~g} / \mathrm{L} \mathrm{MgSO}_{4}$, the average iron oxidation of $310 \mathrm{mg} \mathrm{L}^{-}$ ${ }^{1} \mathrm{~h}^{-1}$ was observed. In contrast, the average iron oxidation rate of starting $L$. ferriphilum reached the maximum of $291 \mathrm{mg} \mathrm{L}^{-1} \mathrm{~h}^{-1}$ at $5 \mathrm{~g} \mathrm{~L}^{-1} \mathrm{MgSO}_{4}$. At pH 0.7, the iron oxidation rate of the evolved $L$. ferriphilum still showed $320 \mathrm{mg} \mathrm{L}^{-1} \mathrm{~h}^{-1}$ at $20 \mathrm{gL}^{-1} \mathrm{MgSO}_{4}$. It was worthy to note that the higher concentration of $\mathrm{MgSO}_{4}$, the smaller the biomass produced by both the evolved strain and the starting strain, indicating that high concentration of $\mathrm{MgSO}_{4}$ is detrimental to the production of biomass, previous studies also demonstrated that high concentration of $\mathrm{Mg}^{2+}$ reduced the biofilm quantity (Tang et al., 2018).Therefore, the enhanced iron oxidation capacity may due to high ionic conductivity environment that improved electron transfer (Li et al., 2014).

The improved tolerance for high concentration $\mathrm{Na}_{2} \mathrm{SO}_{4}$ were also observed (Fig. 5B). However, the iron oxidation rate and biomass decreased gradually with the increase of sodium sulfate concentration. In case of $20 \mathrm{gL}^{-1}$, the iron oxidation rate of the starting strain declined to $250 \mathrm{mg} \mathrm{L}^{-1} \mathrm{~h}^{-1}$, while the iron 
oxidation rate of the evolved strain maintained to $308 \mathrm{mgL}^{-1} \mathrm{~h}^{-1}$. Accordingly, the enhanced performance on acid resistance was beneficial for the improvement of adaptation for sulfate. During bioleaching process, high concentration of sodium sulfate seriously limited the bioleaching efficiency through the formation of Fe(III)-precipitates (Liu et al., 2018) and inhibition effect for microorganisms (Bevilaqua et al., 2013), the adaptation of $L$. ferriphilum in high concentration of sodium sulfate and low pH conditions indicated significant synergetic relationship between acid tolerance and environmental adaptation.

In the absence of organic matter and high $\mathrm{pH}$, the iron oxidation rate of the starting L. ferriphilum was $270 \mathrm{mg} \mathrm{L}^{-1} \mathrm{~h}^{-1}$ and that of the evolved L. ferriphilum was $325 \mathrm{mgL}^{-1} \mathrm{~h}^{-1}$ (Fig $5 \mathrm{C}$ ) However, the iron oxidation rate of the starting strain gradually decreased and only $163 \mathrm{mg} \mathrm{L}^{-1} \mathrm{~h}^{-1}$ was observed under $1 \mathrm{~g} / \mathrm{L}$ organic matter condition. Meanwhile, the total biomass decreased to $0.6 \times 10^{8}$ cells $/ \mathrm{mL}$. These results indicated that the organic matter greatly inhibited the iron oxidation and microbial growth of the starting strain. In contrast, the iron oxidation rate of the evolved strain maintained over $280 \mathrm{mg} \mathrm{L}^{-1} \mathrm{~h}^{-1}$ at same range of organic matter. Especially at low $\mathrm{pH}$ condition, the $310 \mathrm{mg} \mathrm{L}^{-1} \mathrm{~h}^{-1} \mathrm{IOR}$ and $1.7 \times 10^{8}$ cells $/ \mathrm{mL}$ biomass was observed. These results indicated that, although organic compounds still had a strong inhibitory effect on the evolving strains, the performance on adaptation was improved. Therefore, adaptive evolution may be an available strategy to improve adaptation of L. ferriphilum for organic matter. Previous research revealed that the addition of galactose in the medium can significantly improve the adhesion performance of EPS, strengthen the adsorption effect of strain (Aguirre et al., 2018), the evolutionary strain of $L$. ferriphilum increase in sensitivity to organic matter for the application of the bacteria also provides a new train of thought. Besides, no double inhibition effect of organic matter and acidity also revealed the probability of a similar mechanism of tolerance for acidity and organic matter.

Great challenges for bioleaching deriving from the inhibition effect of heavy metals, such as $\mathrm{Ni}, \mathrm{Cu}, \mathrm{Co}$ and $\mathrm{Cr}$ seriously limited iron oxidation and cell yields of microorganism. After adaptation, this phenomenon is still serious (Fig 5D-F). In the case of nickel-containing medium, it was observed that the iron oxidation rate of both the starting strain and the evolving strain declined to $138 \mathrm{mg} \mathrm{L}^{-1} \mathrm{~h}^{-1}$ at $\mathrm{pH} 1.6$, indicating that nickel ion had a serious inhibitory effect on the growth of both the evolving strain and the originating strain, and no great difference in the nickel tolerance was observed between the evolving strain and the starting strain, indicating that only the improvement of acidity tolerance can't realize the adaptation for nickel ion. In contrast, the evolved L. ferriphilum showed $166 \mathrm{mgL}^{-1} \mathrm{~h}^{-1} \mathrm{IOR}$ at $\mathrm{pH} 0.7$. Although nickel ion still has serious influence on the growth of microorganism inhibition, the IOR increased by $24.48 \%$ compared to the high $\mathrm{pH}$ cultivation. The reason for the enhanced nickel ions resistance at low condition may due to acid activated the nickel operon (Tian et al., 2007), implying that the nickel resistance may due to the transcriptional control. In the presence of $\mathrm{Cu}$ and Co ions, it took 72 hours for both the starting strain and the evolved strain to completely oxidize $10 \mathrm{~g} / \mathrm{L}$ ferrous iron at $\mathrm{pH}$ 1.6 , and 96 hours for the evolved strain in $\mathrm{pH} 0.7$. The results indicated the double inhibitory effect of these two metals for $L$. ferriphilum. It is demonstrated the resistance pathway of the strains to these two metals was not consistent with the acid-resisting pathway. No obvious ferrous iron oxidation and biomass increase were observed in the presence of $\mathrm{Cr}$ ions, indicating that $\mathrm{Cr}$ not only inhibited the iron 
oxidation activity of the strain but also inhibited the proliferation of the bacteria. It is concluded that the adaptability to heavy metals was not improved after acid adaptation. Therefore, it is necessary for the improvement of adaptation to heavy metals before the industrial application of acidity adapted $L$. ferriphilum.

\subsection{Bioleaching of metals from PCB and pyrite}

The dissolution of pyrite is observed to vary greatly under bioleaching by starting and evolved strain (Fig. $6 \mathrm{~A})$. At $45^{\circ} \mathrm{C}$, the leaching efficiency of the evolved L. ferriphilum is observed to be $58 \%$ at $\mathrm{pH} 1.6$. In comparison, that of the evolved L. ferriphilum displayed superior pyrite leaching at $\mathrm{pH} 1.0$, the soluble iron achieved for $8.43 \mathrm{~g} / \mathrm{L}(79.52 \%)$. When the temperature of the leach environment was $30^{\circ} \mathrm{C}$, the low $\mathrm{pH}$ leaching performance of the evolved $L$. ferriphilum was inferior to the case of $\mathrm{pH} 1.6$, suggesting that double stress of low temperature and acidity was detrimental to the bioleaching activity of the evolved strain. Meanwhile, the release of iron ion favored the production of acidity (Fig. 6B). All in all, the evolved L. ferriphilum would be advantageous to leach pyrite for the generation of $\mathrm{H}_{2} \mathrm{SO}_{4}$ and $\mathrm{Fe}^{3+}$.

As for PCB bioleaching, no notable difference was observed at $\mathrm{pH} 1.6$ for the cases of the evolved and starting strain (Fig. 6C). The Cu extraction efficiency reached $83 \%$ and $88 \%$, respectively. Similar with pyrite bioleaching, low temperature bioleaching environment showed the lesser metal extraction. Notable observation indicated that bioleaching at low pH seriously inhibited the dissolution of $\mathrm{Cu}$. Our previous studies demonstrated that acid catalyst coupling bioleaching could significantly enhance the $\mathrm{Cu}$ extraction from PCBs(Liu et al., 2020), together revealed the importance of heavy metal resistance for the application of $L$. ferriphilum. After adaptation, the acidity adapted $L$. ferriphilum realized the maximum Cu extraction at pH 1.0 (Fig. 6D), agreeing with previous results that low pH bioleaching eliminated the jarosite barrier and improved the interfacial reaction (Liu et al., 2020). In this regard, it demonstrated that extreme acid tolerance microorganisms would be more promising for the development of bioleaching techniques.

\section{Conclusions}

In this study, L. ferriphilum that could survive in $\mathrm{pH} 0.7$ was obtained from an adaptive evolution community. It showed the enhanced adaptability to acidity, temperature and iron ions. Meanwhile, its growth is slightly affected by the wider range of $9 \mathrm{~K}$ inorganic salt component, and no notable difference in growth was observed at low concentration of ammonium sulfate, dipotassium hydrogen phosphate and potassium chloride. Although the growth of the evolved L. ferriphilum was inhibited by high concentration of organic matter and salt ions to some extent, its tolerance was significantly improved. However, heavy metals still showed a serious inhibitory effect on the growth and iron oxidation of acidity adapted $L$. ferriphilum under the condition of low $\mathrm{pH}$. Compared with starting strain, the evolved $L$. ferriphilum also demonstrated stronger leaching ability before and after adaptation. These findings revealed adaptive evolution of extreme acid tolerance driving the adaptation of acidophiles and thus provided an available approach for the improvement of bioleaching. 


\section{Declarations}

\section{Funding}

This work was supported by the National key R \& D program Task, China (2017YFD0801304). The Heavy Metal Pollution Control Project of WEEE Dismantling Industry in Qingyuan $\triangle$ Phase II \& III $\triangle$ (No. PM-hx020201610-0309).

\section{Competing interests}

Ronghui Liu and Hongbo Zhou declare that they have no conflict of interest.

\section{Availability of data and materials}

All data generated or analyzed during this study are included in this published article.

\section{Authors' contributions}

$\mathrm{RHL}$ carried out the research work and prepared the manuscript. HBZ performed the manuscript preparation and editing of the manuscript.

\section{Consent for publication}

All authors have given their consent to publish this research article.

Acknowledgements

This work was supported by the National key R \& D program Task, China (2017YFD0801304). The Heavy Metal Pollution Control Project of WEEE Dismantling Industry in Qingyuan $\triangle P$ Pase II \& III \ (No. PM-hx020201610-0309).

\section{References}

Aguirre P, Guerrero K, Sanchez-Rodriguez A, Gentina JC, Schippers A (2018) Making sticky cells: effect of galactose and ferrous iron on the attachment of Leptospirillum ferrooxidans to mineral surfaces. Res Microbiol

Baker-Austin C, Dopson M (2007) Life in acid: pH homeostasis in acidophiles. Trends Microbiol 15(4):165-171

Bevilaqua D et al (2013) Effect of Na-chloride on the bioleaching of a chalcopyrite concentrate in shake flasks and stirred tank bioreactors. Hydrometallurgy 138:1-13

Fan X-l et al (2019) Extraction of Al and Ce from coal fly ash by biogenic $\mathrm{Fe}^{3+}$ and $\mathrm{H}_{2} \mathrm{SO}_{4}$. Chem Eng J $370: 1407-1424$ 
Gramp JP, Jones FS, Bigham JM, Tuovinen OH (2008) Monovalent cation concentrations determine the types of Fe(III) hydroxysulfate precipitates formed in bioleach solutions. Hydrometallurgy 94(1-4):29-33

Guan NZ, Liu L (2020) Microbial response to acid stress: mechanisms and applications. Appl Microbiol Biot 104(1):51-65

Li X, Mercado R, Berlinger S, Banta S, West AC (2014) Engineering Acidithiobacillus ferrooxidans Growth Media for Enhanced Electrochemical Processing. Aiche J 60(12):4008-4013

Liu F et al (2018) Significance of jarosite dissolution from the biooxidized pyrite surface on further biooxidation of pyrite. Hydrometallurgy 176:33-41

Liu R, Chen J, Zhou W, Cheng H, Zhou H (2019a) Insight to the early-stage adsorption mechanism of moderately thermophilic consortia and intensified bioleaching of chalcopyrite. Biochem Eng J 144:40-47

Liu R et al (2019b) Enhancing microbial community performance on acid resistance by modified adaptive laboratory evolution. Bioresource Technol 287:121416

Liu R et al (2019c) Selective removal of cobalt and copper from Fe (III)-enriched high-pressure acid leach residue using the hybrid bioleaching technique. J Hazard Mater

Liu RH, Wang W, Zhou WB, Cheng HN, Zhou HB (2020) Acid catalysis coupling bioleaching for enhancement of metals removal from waste resin powder. Journal of Cleaner Production, 247

Ma L, Wang X, Liu X, Wang S, Wang H (2018) Intensified bioleaching of chalcopyrite by communities with enriched ferrous or sulfur oxidizers. Bioresour Technol 268:415-423

Matsumoto M, Ohishi H, Benno Y (2004) H+-ATPase activity in Bifidobacterium with special reference to acid tolerance. Int J Food Microbiol 93(1):109-113

Shiers DW, Collinson DM, Watling HR (2016) Life in heaps: a review of microbial responses to variable acidity in sulfide mineral bioleaching heaps for metal extraction. Res Microbiol 167(7):576-586

Tang D et al., 2018. Mg2 + reduces biofilm quantity in Acidithiobacillus ferrooxidans through inhibiting Type IV pili formation. FEMS Microbiol. Lett., 365(4)

Tian J et al (2007) Nickel-resistant determinant from Leptospirillum ferriphilum. Appl Environ Microb 73(7):2364-2368

Xia MC et al (2017) Recycling of metals from pretreated waste printed circuit boards effectively in stirred tank reactor by a moderately thermophilic culture. J Biosci Bioeng 123(6):714-721

Zhang LJ et al (2015) Synergetic effects of Ferroplasma thermophilum in enhancement of copper concentrate bioleaching by Acidithiobacillus caldus and Leptospirillum ferriphilum. Biochem Eng $\mathrm{J}$ 93:142-150 
Zhang RY et al (2010) A new strain Leptospirillum ferriphilum YTW315 for bioleaching of metal sulfides ores. T Nonferr Metal Soc 20(1):135-141

Zhou D et al (2015) Expression of Critical Sulfur- and Iron-Oxidation Genes and the Community Dynamics During Bioleaching of Chalcopyrite Concentrate by Moderate Thermophiles. Curr Microbiol 71(1):62-69

Zhou WB et al (2019) Cleaner utilization of electroplating sludge by bioleaching with a moderately thermophilic consortium: A pilot study. Chemosphere 232:345-355

\section{Figures}

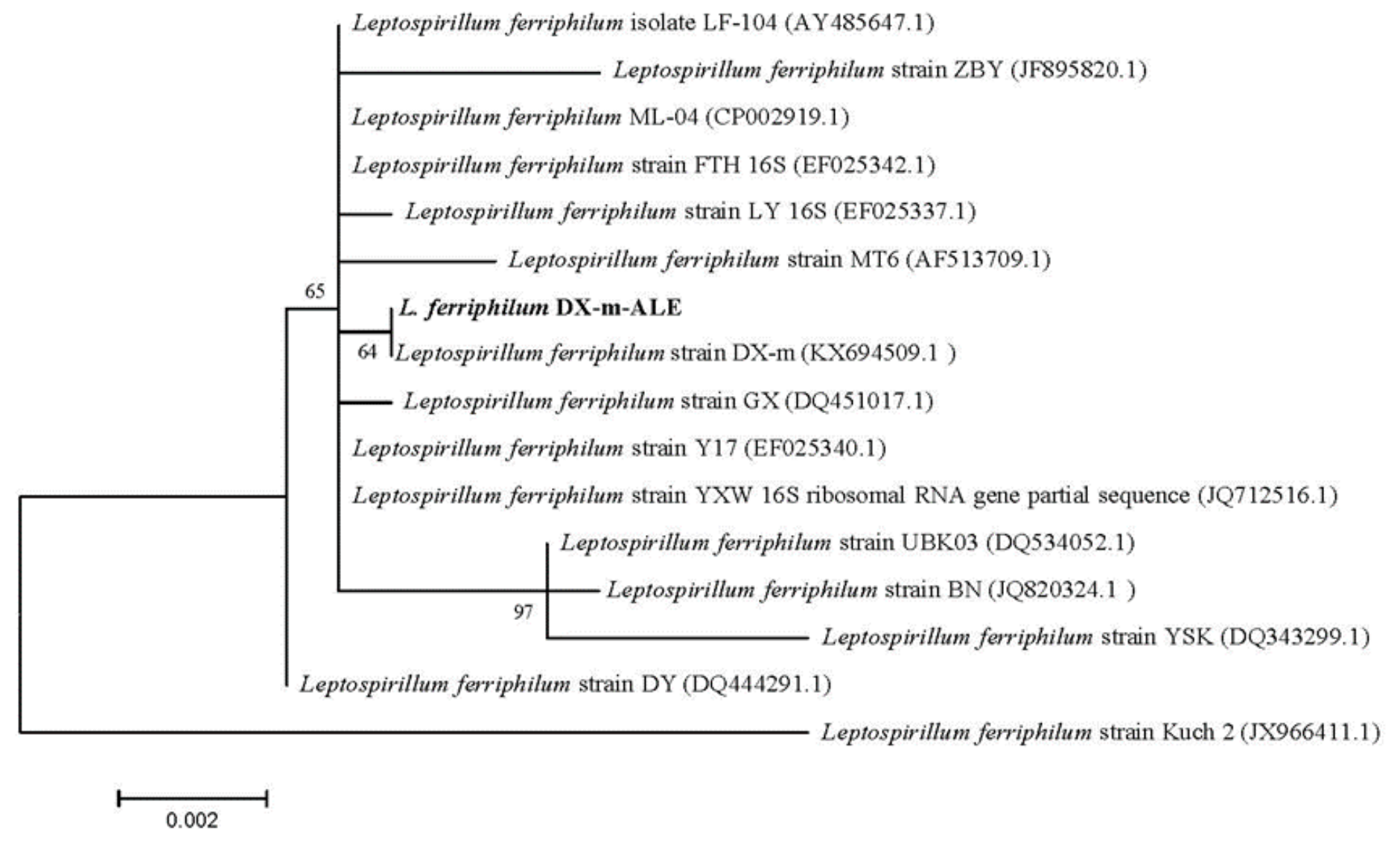

\section{Figure 1}

Phylogenetic tree analysis of the isolated L. ferriphilum 


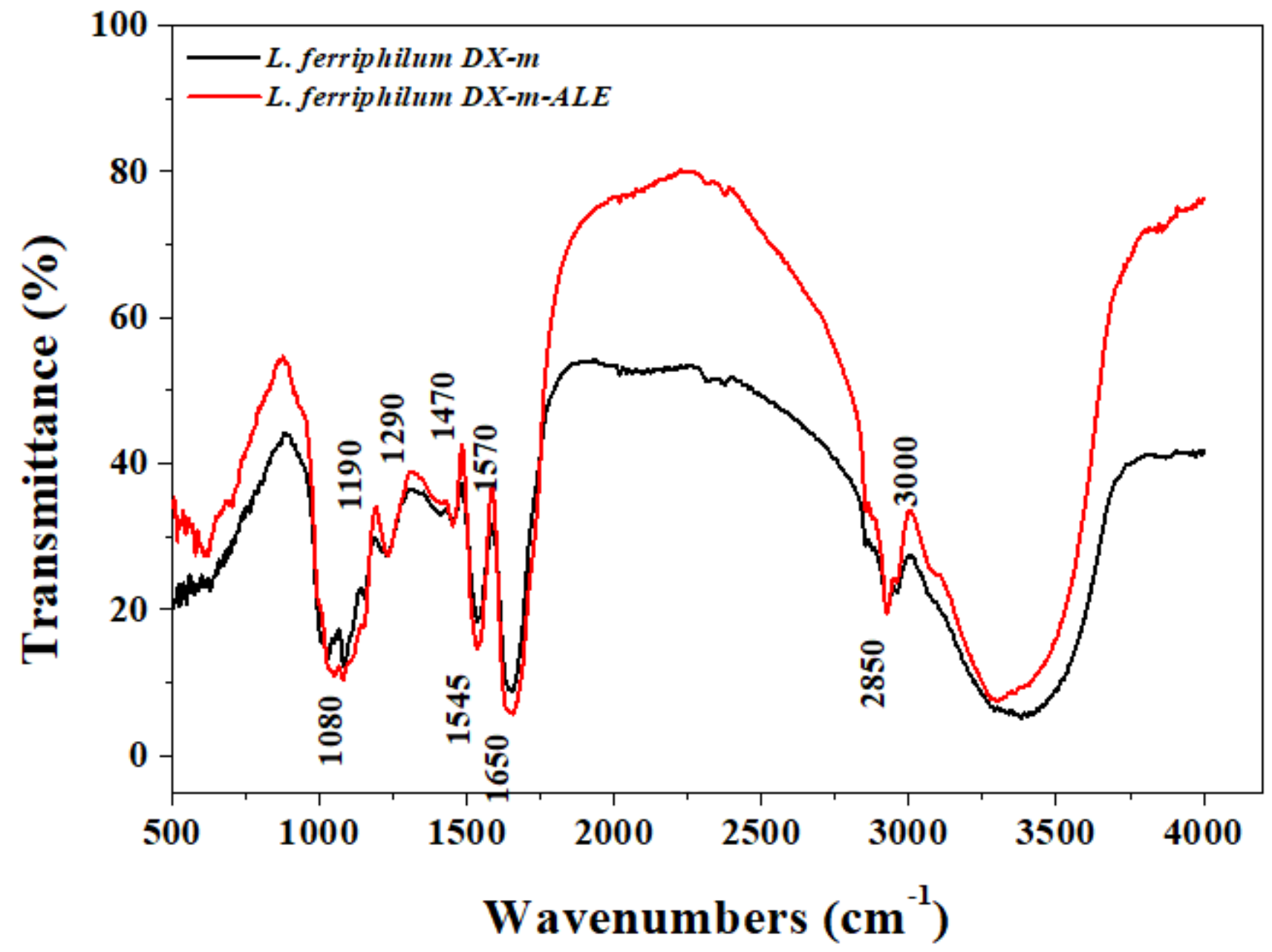

Figure 2

FTIR analysis of L. ferriphilum DX-m and L. ferriphilum DX-m-ALE 

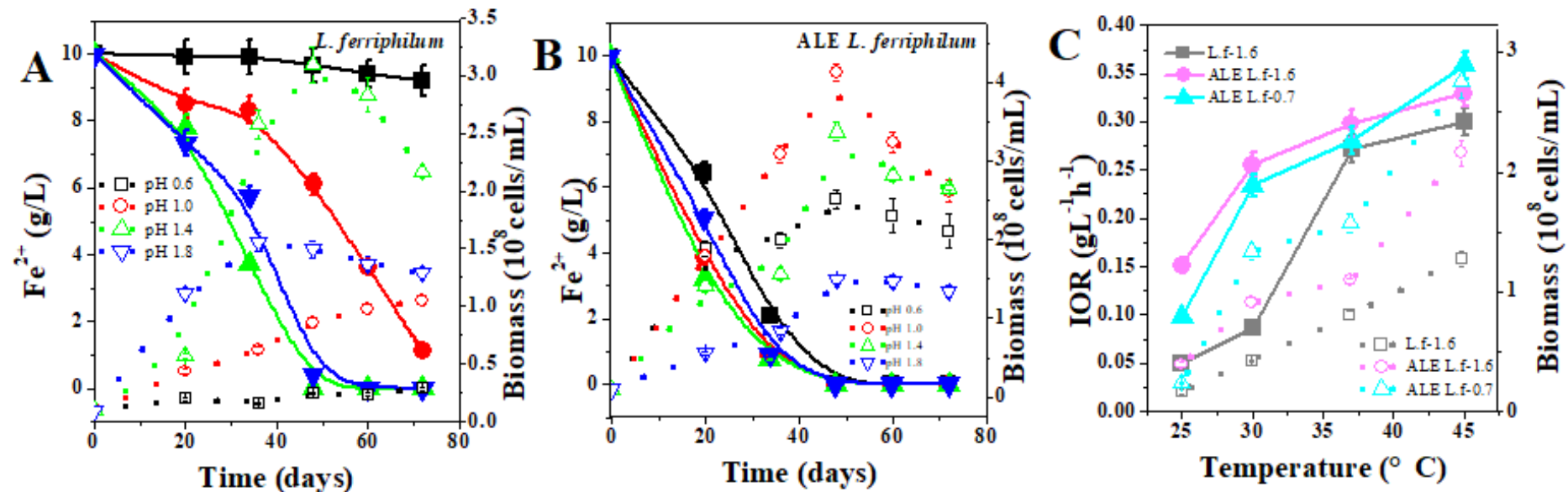

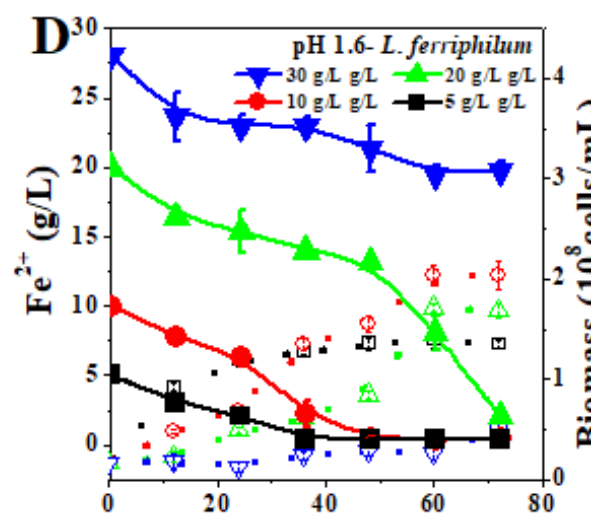

Time (hours)

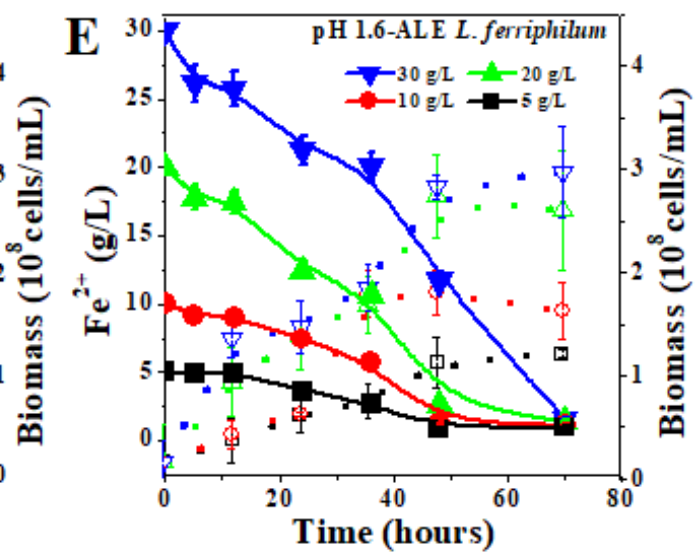

Time (hours)

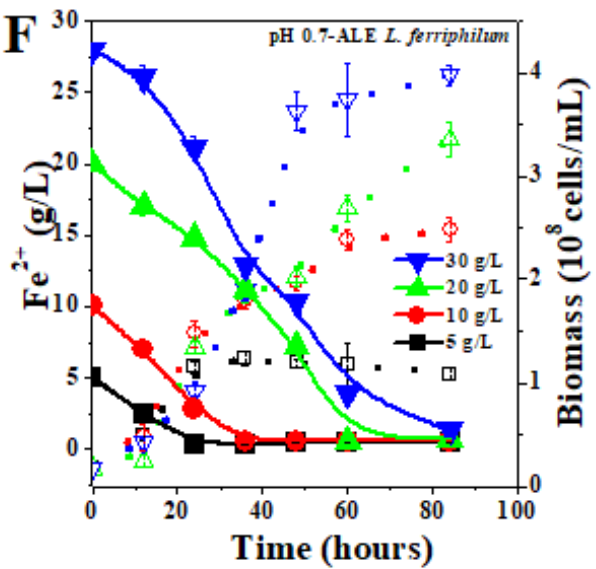

\section{Figure 3}

The growth of starting and evolved strain under different biochemical factors condition (Solid: Fe2+ or IOR; Open: Biomass; A: Culturing L. ferriphilum DX-m at different pH value; B: Culturing L. ferriphilum DXm-ALE at different $\mathrm{pH}$ value; C: IOR and biomass production for $L$. ferriphilum DX-m and L. ferriphilum DX-m-ALE at different temperature (L.f 1.6: Culturing L. ferriphilum DX-m at pH 1.6; ALE L.f-1.6: Culturing L. ferriphilum DX-m-ALE at pH 1.6; ALE L.f-0.7: Culturing L. ferriphilum DX-m-ALE at pH 0.7); D: IOR and biomass production for L. ferriphilum DX-m at pH 1.6 and different concentration of ferrous iron; E: IOR and biomass production for L. ferriphilum DX-m-ALE at pH 1.6 and different concentration of ferrous iron; F: IOR and biomass production for L. ferriphilum DX-m-ALE at pH 0.7 and different concentration of ferrous iron) 

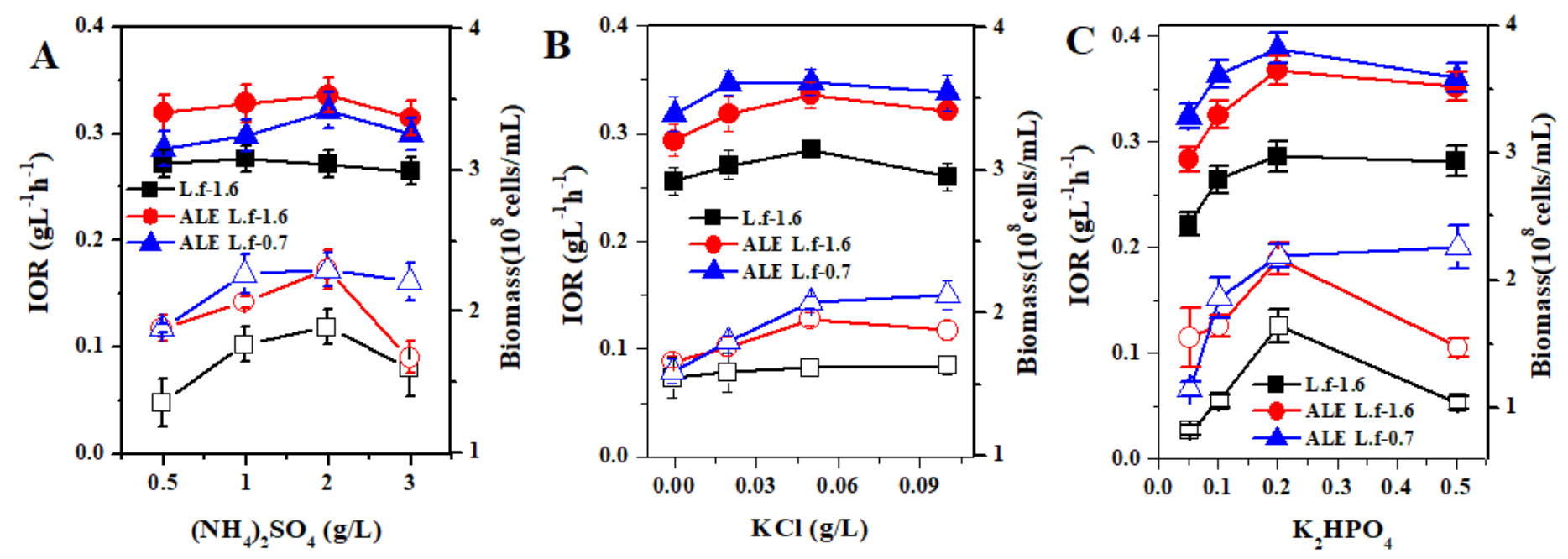

Figure 4

Iron oxidation capacity and biomass production of starting and evolved strain under different concentrations of $9 \mathrm{~K}$ basic salt (Solid: IOR; Open: Biomass; L.f 1.6: Culturing L. ferriphilum DX-m at pH 1.6; ALE L.f-1.6: Culturing L. ferriphilum DX-m-ALE at pH 1.6; ALE L.f-0.7: Culturing L. ferriphilum DX-mALE at pH 0.7; A: (NH4)SO4; B: KCl; C: K2HPO4) 

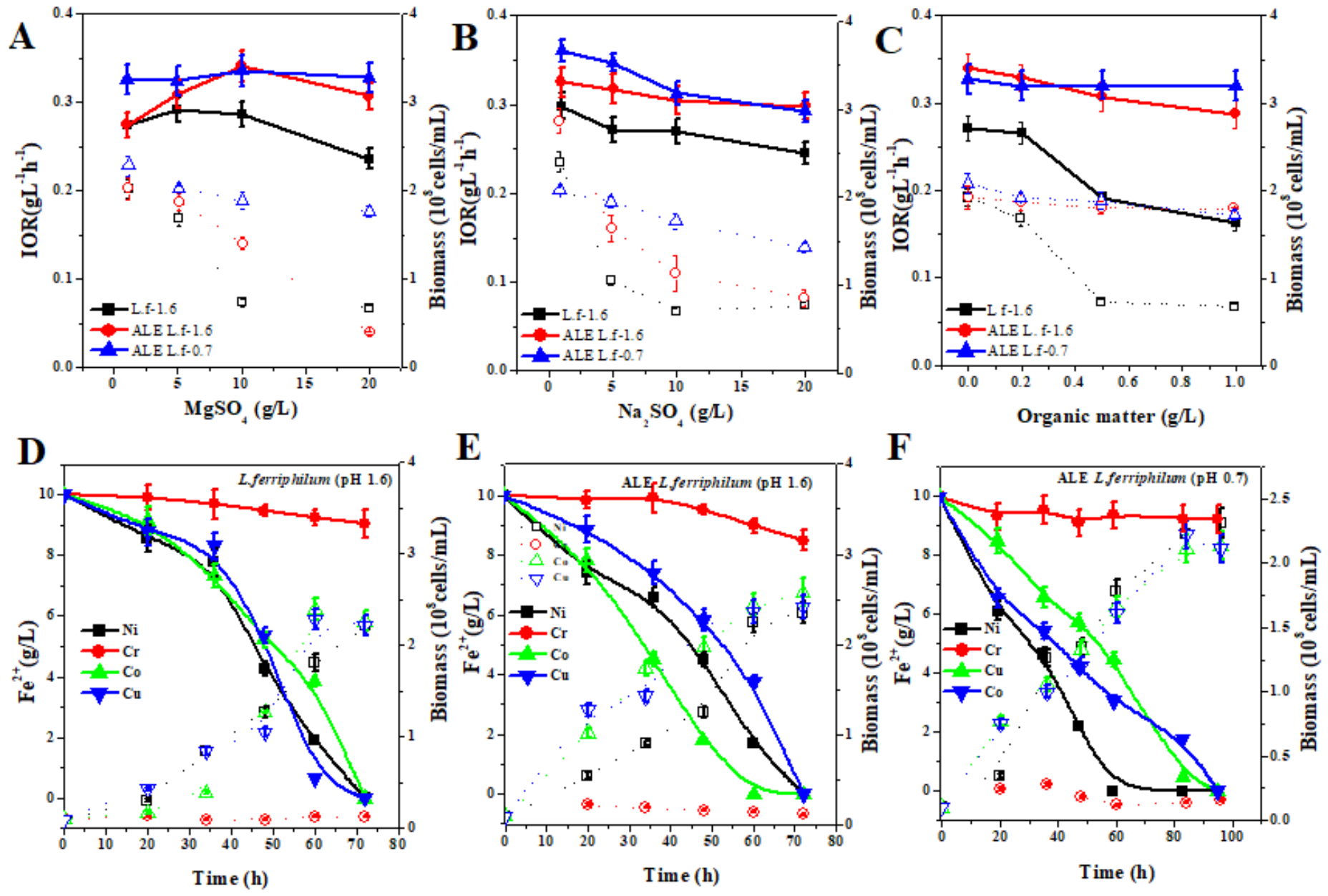

\section{Figure 5}

Iron oxidation capacity and biomass production of starting and evolved strain under high concentrations salt, organic matter and heavy metals conditions (Solid: IOR or Fe2+; Open: Biomass; A: MgSO4; B: Na2SO4; C: organic matter; D: The culture of L. ferriphilum DX-m with heavy metals at pH 1.6; E: The culture of L. ferriphilum DX-m-ALE with heavy metals at pH 1.6; F: The culture of L. ferriphilum DX-m-ALE with heavy metals at $\mathrm{pH}$ 0.7) 

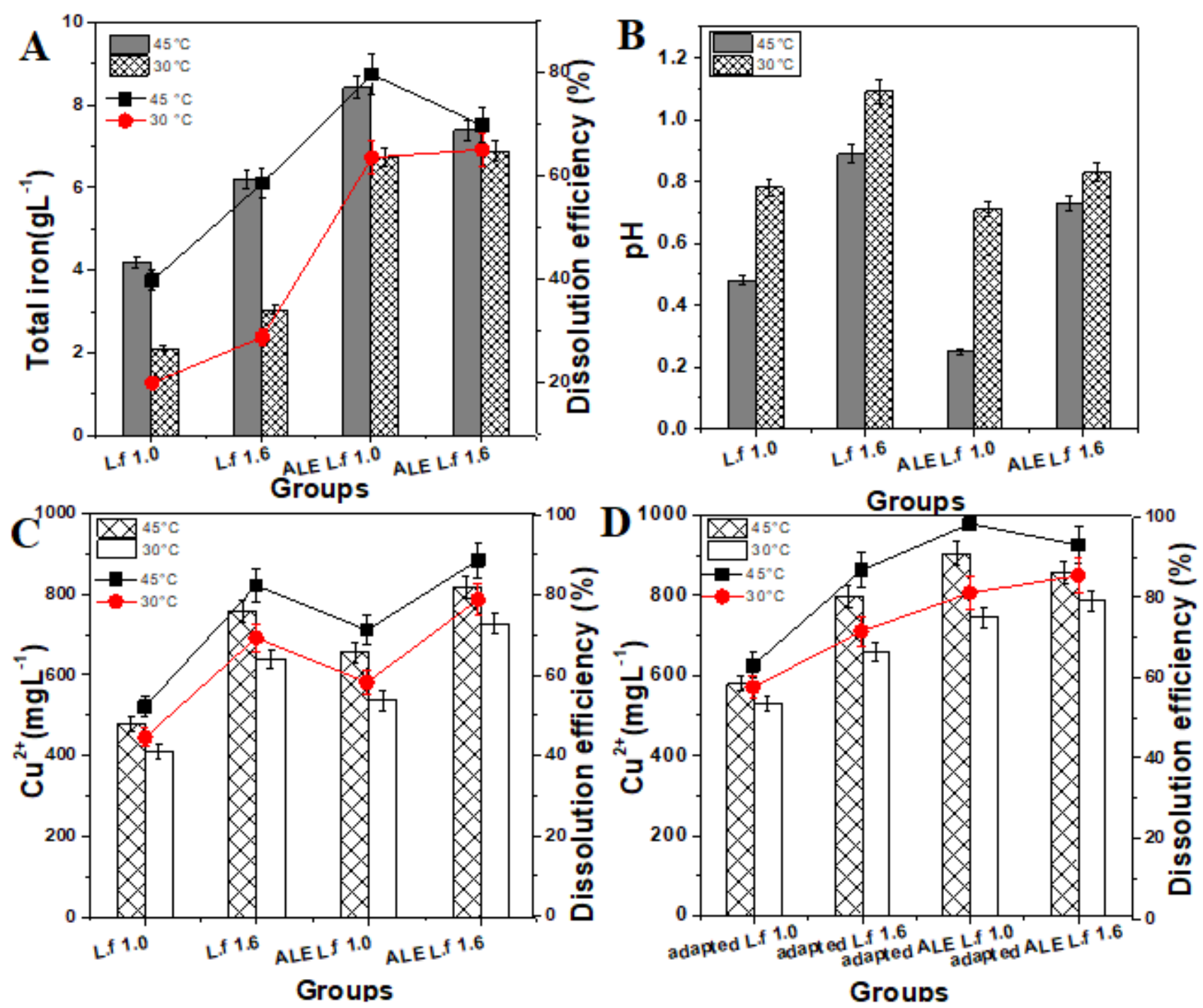

Figure 6

the biochemical parameters during pyrite and PCB bioleaching process (Colum: metals concentration; Line: dissolution efficiency; $\mathrm{A}$ : the extracted total iron from pyrite. $\mathrm{B}$ : Changes of $\mathrm{Ph}$ during pyrite bioleaching; C: PCB bioleaching by L. ferriphilum DX-m and L. ferriphilum DX-m-ALE; D: PCB bioleaching by adapted L. ferriphilum DX-m and adapted L. ferriphilum DX-m-ALE) 